\title{
Overexpression of Tiam1 promotes the progression of laryngeal squamous cell carcinoma
}

\author{
SHUANG WANG ${ }^{*}$, SHISHENG LI* ${ }^{*}$, QINGLAI TANG, SHU YANG, SHUHUI WANG, \\ JIAJIA LIU, MI YANG and XINMING YANG \\ Department of Otolaryngology, Head and Neck Surgery, The Second Xiangya Hospital, \\ Central South University, Changsha, Hunan 410011, P.R. China
}

Received December 3, 2014; Accepted January 21, 2015

DOI: 10.3892/or.2015.3785

\begin{abstract}
T-lymphoma invasion and metastasis-inducing factor 1 (Tiam1) has been reported in various types of human cancer, which play important roles in facilitating the metastasis of malignant tumor. However, the investigation of Tiam1 in laryngeal squamous-cell carcinoma is extremely rare. The aim of the present study was to assess Tiam1 expression and examine its function in tumorigenesis and the metastasis of laryngeal squamous cell carcinoma (LSCC) in vitro. Tiam1 expression in 98 primary LSCC tissue specimens was analyzed by immunohistochemistry and correlated with clinicopathological parameters and patients' survival. To investigate the effects of Tiam1 on the progression of LSCC, Tiam1/C1199 plasmid was transfected into LSCC, and proliferation, apoptosis, migration and invasion of transfected cells were examined using MTT, flow cytometry, wound-healing and Transwell assay, respectively. The results showed that, Tiam1 was detected in all primary LSCC samples. Additionally, Tiam 1 overexpression was closely correlated with tumor progression and patient survival. Tiam1 overexpression was statistically significant, and served as an independent predictor of prognosis for patients with LSCC. The upregulation of Tiam1 by Tiam1/C1199 plasmid had no effect on the proliferation of transfected cells, but decreased the apoptotic rate of transfected cells, while the ability of migration and invasion was increased. These results suggested that Tiam1 overexpression in LSCC is possibly involved in the promotion of migration and invasion, and is a promising therapeutic target in the prevention of the progression of LSCC.
\end{abstract}

Correspondence to: Dr Xinming Yang, Department of Otolaryngology, Head and Neck Surgery, The Second Xiangya Hospital, Central South University, 139 Renmin Road, Changsha, Hunan 410011, P.R. China

E-mail: x16y2013@outlook.com

*Contributed equally

Key words: invasion, laryngeal squamous-cell carcinoma, metastasis, prognosis, Tiam1

\section{Introduction}

Laryngeal squamous cell carcinoma (LSCC) is a common tumor occurring in the head and neck, and accounts for $~ 5 \%$ of all cancer cases (1). Despite advances in the development of conventional therapies such as surgery, radiotherapy and chemotherapy, the 5-year survival rate for patients with LSCC remains unsatisfactory ( $\sim 50-70 \%)$ (2). Locoregional recurrences, lymph node and distant metastasis are major causes of death that significantly affect prognosis in LSCC patients (3). Therefore, the recognition and identification of tumor markers associated with recurrence and/or metastasis is of great value in the prediction of malignant biological behavior and the direction of therapeutic strategies.

T-lymphoma invasion and metastasis-inducing factor 1 (Tiam1), as an invasion and metastasis-inducing gene, was first identified by proviral tagging in combination with in vitro selection for invasiveness from a murine T-lymphoma cell line (4). The Rho-like small guanosine triphosphate (GTP)-binding proteins, as molecular switches, cycle between an inactive and an active GTP-bound state. Guanine nucleotide exchange factors (GEFs), which catalyze the exchange of GDP for GTP, positively stimulte these GTP-binding proteins in response to various signaling pathways (5). Tiam1, as a member of Db1 family proteins, is a specific GEF for the Rho-like GTP-binding protein Rac, which is involved in cell migration, invasion and metastasis (6).

Tiam1 overexpression is prevalent in numerous solid tumors, including those of prostate (7), hepatocellular $(8,9)$, nasopharyngeal (10), esophageal (11) and renal cell carcinoma (12), colorectal cancer (13) and gallbladder carcinoma (14). Tiam1 is also reported to have intimate correlations with apoptosis (15), invasion and migration $(16,17)$. Therefore, the overexpression of Tiam1 is important in the progression of malignant tumors. However, to the best of our knowledge, few studies evaluating the role of Tiam1 protein expression in LSCC are available. Consequently, we investigated Tiam 1 expression in a number of LSCC tissue samples and assessed whether Tiam1 expression was correlated with clinicopathological parameters and prognosis in LSCC patients. Moreover, Tiam1/C1199 plasmid was employed to upregulate Tiam1 expression in LSCC cell lines, and the roles of Tiam1 in LSCC cell growth, apoptosis, migration, and invasion in vitro were investigated. 


\section{Materials and methods}

Tissue samples and patients. A total of 98 patients with LSCC identified in the Department of Otolaryngology Head and Neck Surgery of Second Xiangya Hospital in Central South University from January 2003 to December 2006 were studied retrospectively. All 98 included patients had no history of radiotherapy or chemotherapy. All patients received treatment with curative intent and definitive resection and/ or therapeutic comprehensive neck dissection, and/or postsurgical radiotherapy or chemotherapy. After the completion of treatment, the patients underwent routine surveillance every 1-3 months. Four patients were lost to follow up due to home moving or telephone number changes. Recurrence and metastasis were determined by physical examination, imaging evaluation, operation and postoperative pathological examinations. Overall and disease-free survival were calculated from the day of surgery to the day the patient succumbed or that of tumor relapse. The main clinical and pathological variables of the 98 patients are described in detail in Table I. The patients involved in the present study provided written informed consent. The investigation was approved by the Research Ethics Committee of Central South University, Changsha, China.

Immunohistochemistry. Immunohistochemical staining was performed using the PV-6001 Two-Step IHC Detection reagent following the manufacturer's instructions (Zhongshan Golden Bridge Biotechnology, Beijing, China). Briefly, antigen retrieval was carried out in $10 \mathrm{mmol} / \mathrm{l}$ citrate buffer $(\mathrm{pH} 6.0)$ for $10 \mathrm{~min}$ in a microwave oven at $750 \mathrm{~W}$. Endogenous peroxidase activity was blocked with $3 \%$ hydrogen peroxide for $15 \mathrm{~min}$ at room temperature. Slides were incubated with Tiam1 rabbit polyclonal antibody (sc-872, dilution 1:50) (Santa Cruz Biotechnology Inc., Santa Cruz, CA, USA) at $4^{\circ} \mathrm{C}$ overnight, followed by the addition of HRP-labelled goat anti-rabbit polymers. Immunoreactive proteins were visualised with 3,3'-diaminobenzidine and counterstained with Mayer's hematoxylin. Negative control slides were probed with phosphate-buffered saline (PBS) under the same experimental conditions. The sections were independently evaluated and scored by two pathologists who were blinded to the clinical data of the patients. In case of disagreement, the sections were reviewed by a third person until a consensus conclusion was reached. The staining intensity was scored as 0 (no staining), 1 (weakly stained, light yellow), 2 (moderately stained, yellowish brown) and 3 (strongly stained, brown). The extent of staining was scored as 0 (absent), 1-5\% (sporadic), 6-25\% (local), $26-50 \%$ (occasional), $51-75 \%$ (majority) and $76-100 \%$ (large majority), according to the percentage of positively stained cells in the entire carcinoma-involved area. An intensity score of $\geq 2$ with $\geq 50 \%$ of malignant cells with positive Tiam 1 staining was considered high expression, and a $<2$ intensity score or $<50 \%$ of malignant cells with positive staining was considered to be low expression of Tiam1 (12).

Cell culture and stable transfection. The human Hep-2 LSCC cell line, purchased from the Type Culture Collection of the Chinese Academy of Sciences, Shanghai, China, was used in the present study. The cell line was grown in RPMI-1640 medium (Invitrogen, Carlsbad, CA, USA) supplemented with $10 \%$ fetal bovine serum (HyClone, Logan, UT, USA), 100 IU/ $\mathrm{ml}$ penicillin and $100 \mathrm{IU} / \mathrm{ml}$ streptomycin, and maintained in a humidified atmosphere of $5 \% \mathrm{CO}_{2}$ at $37^{\circ} \mathrm{C}$. The Tiam1 cDNA (C1199) plasmid cloned as a BamHI/XhoI fragment in pcDNA3 containing a cytomegalovirus promoterand ahemagglutinintag, a generous gift provided by Dr John Collard (The Netherlands Cancer Institute, Amsterdam, The Netherlands), was used for stable transfection. Hep- 2 cells $\left(3 \times 10^{5}\right.$ cells/well) were seeded into 6-well plates and allowed to grow at $50-70 \%$ confluence. The cells were transfected with Tiam1/C1199 plasmid and vector control using Lipofectamine 2000 (Invitrogen), according to the manufacturer's instructions. After $8 \mathrm{~h}$, the original medium was replaced with fresh complete medium and the cells were subjected to the selection of stable clones in the presence of G418 $(800 \mu \mathrm{g} / \mathrm{ml})$ (Amresco, Solon, OH, USA) $48 \mathrm{~h}$ post-infection. The expression of Tiam1 was determined by western blotting after 3 weeks of G418 selection in RPMI-1640 medium containing 10\% fetal bovine serum.

Western blotting. Total protein lysates, harvested from cells, were quantified by Bicinchoninic Acid Protein Assay kit (Beyotime, China). Total protein ( $40 \mu \mathrm{g}$ ) was separated by 8\% SDS-PAGE and then transferred onto polyvinylidene difluoride membranes (Immobilon-P; Millipore, Bedford, MA, USA). The membranes were incubated with rabbit polyantibody against Tiam1 (sc-872, dilution 1:100) (Santa Cruz Biotechnology Inc.) at $4^{\circ} \mathrm{C}$ overnight. After washing, the cells were incubated with HRP-labelled goat anti-rabbit $\operatorname{IgG}$ for $2 \mathrm{~h}$ at room temperature. Bands were finally visualized by employing 3,3'-diaminobenzidine (Zhongshan Golden Bridge Biotechnology). Tiam1 protein expression levels were quantified by Quantity One software (Bio-Rad, Hercules, CA,USA). $\beta$-actin protein was determined using the specific antibody (BA2350, dilution 1:500) (Boster, China) as a loading control. All the experiments were carried out in triplicate.

MTT cell viability assay. The MTT assay was performed to evaluate the proliferation of transfected cells. The cells $\left(5 \times 10^{3}\right.$ cells/well) were plated in three 96-well plates in $200 \mu \mathrm{l}$ of medium, and exposed to fresh media on alternate days. At 1,2 and 3 day after plating, $20 \mu \mathrm{l}$ MTT (5 mg/ml) (Sigma) was added to each well of one plate and after $4 \mathrm{~h}$, the liquids were removed and $150 \mu 1$ dimethyl sulfoxide (DMSO) was added. The optical density (OD) of each well was measured at $570 \mathrm{~nm}$ with a microplate reader. The cell growth curves were drawn according to time (x-axis) and OD value (y-axis).

Cell cycle analysis and cell apoptosis by flow cytometry. For cell cycle analysis, cells $\left(1 \times 10^{6} / \mathrm{ml}\right)$ were washed with PBS at $4^{\circ} \mathrm{C}$ twice and fixed in $70 \%$ alcohol for $24 \mathrm{~h}$. Cell apoptosis was examined by flow cytometry using an Annexin V-FITC apoptosis detection kit (Invitrogen), following the manufacturer's instructions. The cells were washed twice in ice PBS and adjusted to a concentration of $1 \times 10^{6} / \mathrm{ml}$. The cells were subsequently gently mixed with $5 \mu 1$ Annexin V-FITC and $5 \mu \mathrm{l}$ propidium iodide (PI), and incubated for $15 \mathrm{~min}$ at room temperature in the dark. After the addition of another $400 \mu \mathrm{l}$ of $1 \mathrm{X}$ binding buffer, cell sorting analysis was detected on a FACSCalibur cytometer (Becton-Dickinson). 
Cell migration and invasion assay. For the cell migration assay, the wound-healing assay was carried out. The cells $\left(5 \times 10^{5}\right.$ cells/well) were seeded in 6 -well plates with complete medium and incubated for $12 \mathrm{~h}$ to until $90 \%$ confluence was reached. The cell monolayer was scratched with a $10 \mu \mathrm{l}$ pipette tip, images were captured at 0 and $48 \mathrm{~h}$ after disruption in a phase-contrast microscope. Data presented are representative of three individual experiments.

The cell invasion assay was evaluated using 24-well Transwell chambers (Costar, Cambridge, MA, USA), according to the manufacturer's instructions. Briefly, Transwell with an $8-\mu \mathrm{m}$ pore size insert membrane was coated with $100 \mu \mathrm{l}$ Matrigel (BD Biosciences, Bedford, MA, USA) and incubated overnight. The cells $\left(3 \times 10^{4}\right.$ cells/well) in $100 \mu \mathrm{l}$ of serum-free medium were seeded in the upper chamber of the Transwell, while the lower chamber was filled with $0.6 \mathrm{ml}$ RPMI-1640 medium containing $15 \%$ fetal bovine serum to induce chemotaxis. After $24 \mathrm{~h}$ of incubation at $37^{\circ} \mathrm{C}$ in humidified $5 \% \mathrm{CO}_{2}$ atmosphere, the non-migratory cells on the upper member surface were removed with a cotton tip, the migrated cells were fixed in $95 \%$ alcohol and stained with hematoxylin, and the number of migrated cells was counted in five randomly selected x400 power fields under the microscope. Results presented are representative of three individual wells.

Statistical analysis. Correlations between the expression of Tiam1 protein and clinicopathological parameters were calculated using the $\chi^{2}$ test. Survival analysis was undertaken using the Kaplan-Meier method and curves were compared by the log-rank test. The Cox proportional hazards regression model was used in univariate and multivariate analysis to assess which factors were independent indicators for prognosis. Continuous variables were presented as mean \pm SD. One-way ANOVA was used to compare the differences between the three cell groups. The SPSS 17.0 software package (SPSS, Inc., Chicago, IL, USA) was used for statistical analyses. $\mathrm{P}<0.05$ was considered to indicate a statistically significant result.

\section{Results}

Tiaml expressionin98casesofLSCCbyimmunohistochemistry. Positive Tiam1 immunostaining was predominantly diffusely distributed throughout the cytoplasm of tumor cells (Fig. 1). Among all the samples analyzed, 32 (32.7\%) and $66(67.3 \%)$ cases demonstrated a high and low Tiam1 protein expression. The $\chi^{2}$ test was applied to assess the association between Tiam1 protein levels and various clinicopathological variables. As shown in Table I, Tiam1 overexpression was significantly associated with lymph-node metastasis $(\mathrm{P}<0.001)$, clinical stage $(\mathrm{P}=0.027)$, histological grade $(\mathrm{P}=0.020)$, and recurrence $(\mathrm{P}=0.003)$, respectively. However, no significant relationship was found between Tiam1 protein level and variables such as age, primary site and $\mathrm{T}$ stage (all $\mathrm{P}>0.05$ ). According to the Tiam1 protein expression levels, 94 patients with follow-up (4 patients lost) were subdivided into two groups: 63 patients in the low Tiam1 expression group and 31 patients in the high Tiam 1 expression group. The disease-free and overall survival rate in the 94 patients was 57.4 and $63.8 \%$, respectively. Tiam1 expression in LSCC was significantly correlated with diseasefree survival $(\mathrm{P}=0.001$; Fig. $2 \mathrm{~A})$ and overall survival $(\mathrm{P}<0.001$;
Table I. Correlations between Tiam1 expression and clinicopathological parameters in patients with laryngeal squamous cell carcinoma.

\begin{tabular}{|c|c|c|c|c|}
\hline \multirow[b]{2}{*}{$\begin{array}{l}\text { Clinicopatholo- } \\
\text { gical variables }\end{array}$} & \multirow[b]{2}{*}{$\begin{array}{l}\text { No. of } \\
\text { pts. }\end{array}$} & \multicolumn{2}{|c|}{ Tiam1 expression } & \multirow[b]{2}{*}{ P-value ${ }^{a}$} \\
\hline & & $\begin{array}{c}\text { Low } \\
\text { expression }\end{array}$ & $\begin{array}{c}\text { High } \\
\text { expression }\end{array}$ & \\
\hline Age (years) & & & & 0.139 \\
\hline$<58$ & 41 & 31 & 10 & \\
\hline$\geq 58$ & 57 & 35 & 22 & \\
\hline Primary site & & & & 0.935 \\
\hline Glottic & 74 & 50 & 24 & \\
\hline Supraglottic & 24 & 16 & 8 & \\
\hline T stage & & & & 0.857 \\
\hline $\mathrm{T} 1+\mathrm{T} 2$ & 60 & 40 & 20 & \\
\hline $\mathrm{T} 3+\mathrm{T} 4$ & 38 & 26 & 12 & \\
\hline $\begin{array}{l}\text { Lymph node } \\
\text { metastasis }\end{array}$ & & & & $<0.001$ \\
\hline N0 & 76 & 58 & 18 & \\
\hline $\mathrm{N}+(\mathrm{N} 1, \mathrm{~N} 2, \mathrm{~N} 3)$ & 22 & 8 & 14 & \\
\hline $\begin{array}{l}\text { Clinical stage } \\
\text { (TNM) }\end{array}$ & & & & 0.027 \\
\hline I-II & 40 & 32 & 8 & \\
\hline III-IV & 58 & 34 & 24 & \\
\hline Histological grade & & & & 0.020 \\
\hline $\mathrm{G} 1$ & 59 & 45 & 14 & \\
\hline $\mathrm{G} 2+\mathrm{G} 3$ & 39 & 21 & 18 & \\
\hline Recurrence $^{\mathrm{b}}$ & & & & 0.003 \\
\hline Yes & 40 & 20 & 20 & \\
\hline No & 54 & 43 & 11 & \\
\hline
\end{tabular}

${ }^{\mathrm{a}} \mathrm{P} \leq 0.05$ was considered to be statistically significant (in bold). ${ }^{\mathrm{b}}$ Four patients were lost to follow up due to home moving or telephone number change. pts., patients; TNM, tumor-node-metastasis.

Fig. 2B). The log-rank test also demonstrated that the survival time was significantly different between groups with a high and low expression of Tiam1 protein, indicating that a high level of Tiam1 was correlated with a shorter survival time. The Cox proportional hazards regression model was used to assess the effect of clinicopathological parameters and Tiam1 expression on the disease prognosis. In the univariate Cox analysis, lymph-node metastasis, clinical stage and Tiam1 expression were statistically correlated with prognosis. The multivariate Cox proportional hazards model analysis confirmed that lymph-node metastasis and Tiam1 expression were statistically significant, independent prognostic factors of outcomes in LSCC patients (Table II).

Upregulated expression of Tiaml by plasmid Tiam1/C1199 cDNA in Hep-2 cells. To clarify the correlation of Tiam1 expression and LSCC metastasis, we employed the Tiam1/C1199 cDNA plasmid to improve the expression of Tiam1. Stable clones were isolated after selection with $800 \mu \mathrm{g} / \mathrm{ml} \mathrm{G} 418$ for 

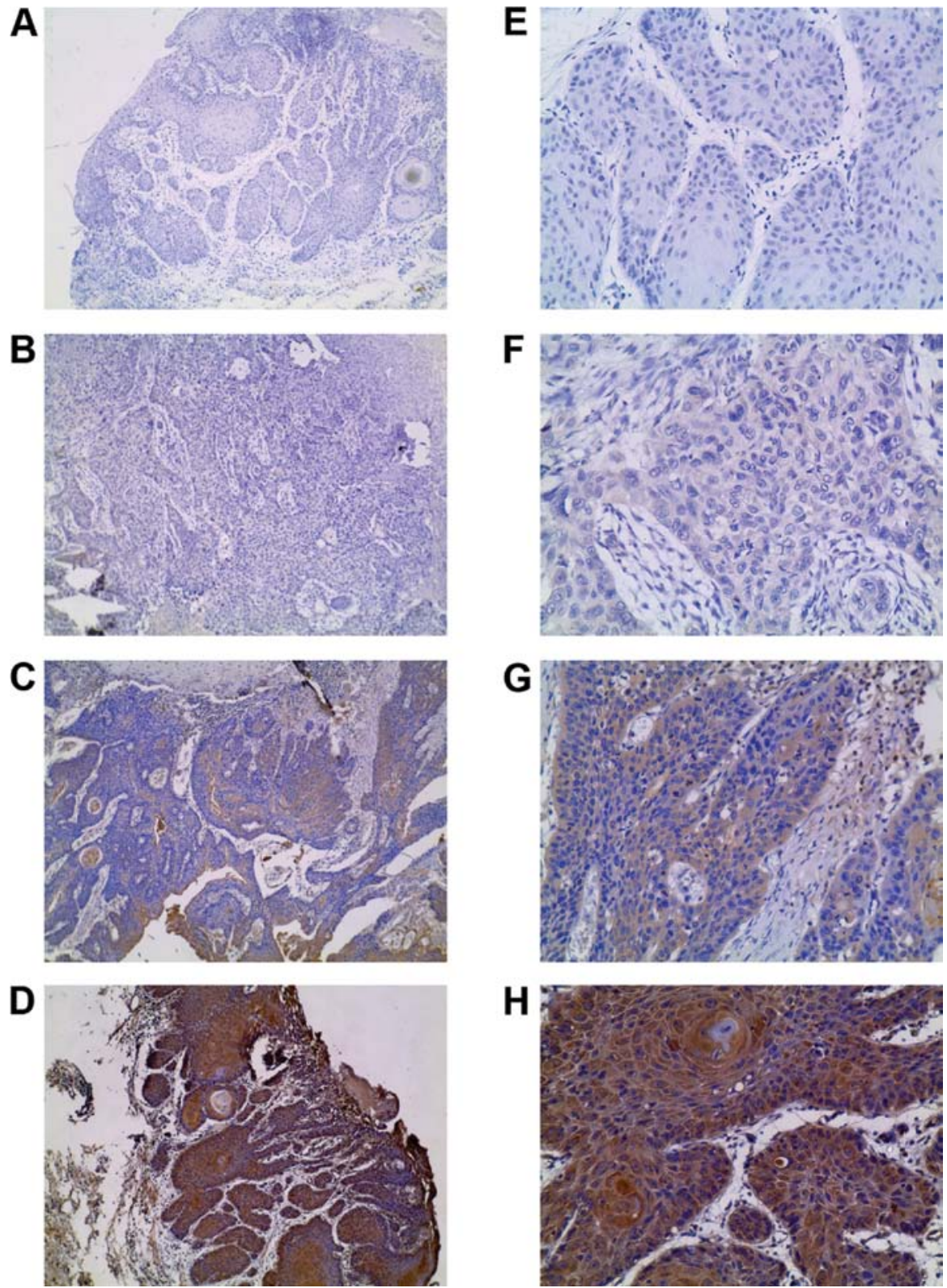

Figure 1. Immunohistochemistry of Tiam1 in human laryngeal squamous-cell carcinoma (LSCC) tissues. (A and E) Negative control of Tiam1 in LSCC tissues (primary antibody replaced with PBS). (B and F) Weak staining of Tiam1 in LSCC tissues. (C and G) Moderate staining of Tiam1 in LSCC tissues. (D and H) Strong staining of Tiam1 in LSCC tissues. (Original magnification, x100 in A-D, and x400 in E-H). Tiam1, T-lymphoma invasion and metastasis-inducing factor 1; PBS, phosphate-buffered saline.
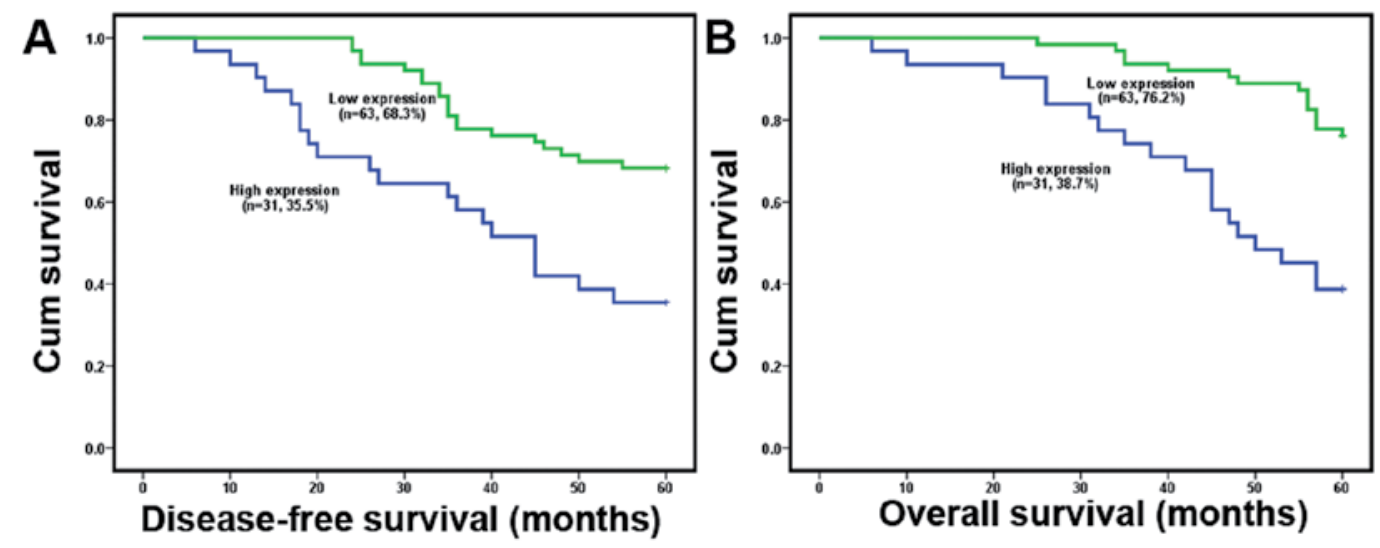

Figure 2. Kaplan-Meier survival curves showing (A) 5-year disease-free and (B) overall survival rate of patients with a high or low Tiam1 expression. Log-rank test demonstrated that patients with laryngeal squamous-cell carcinoma in the high Tiam1 expression group had a shorter disease-free survival rate $(\mathrm{P}=0.001)$ and poorer overall survival rate $(\mathrm{P}<0.001)$ compared with those in the low Tiam1 expression group. Tiam1, $\mathrm{T}-\mathrm{lymphoma}$ invasion and metastasis-inducing factor 1 . 
Table II. Cox proportional hazards regression model analysis of overall survival.

\begin{tabular}{|c|c|c|c|c|}
\hline \multirow[b]{2}{*}{ Clinicopathological variables } & \multicolumn{2}{|c|}{ Univariate analysis } & \multicolumn{2}{|c|}{ Multivariate analysis } \\
\hline & $\operatorname{Exp}(B)$ & P-value & $\operatorname{Exp}(B)$ & P-value \\
\hline Age (years) $(<58 / \geq 58)$ & 1.746 & 0.129 & 0.624 & 0.300 \\
\hline Primary site (glottic/subglottic) & 1.010 & 0.980 & 1.089 & 0.833 \\
\hline $\mathrm{T}$ stage $(\mathrm{T} 1+\mathrm{T} 2 / \mathrm{T} 3+\mathrm{T} 4)$ & 0.489 & 0.066 & 3.424 & 0.142 \\
\hline Lymph node metastasis $(\mathrm{N} 0 / \mathrm{N}+)$ & 6.757 & $<0.001$ & 29.241 & 0.001 \\
\hline Clinical stage (I-II/III-IV) & 1.315 & 0.044 & 0.145 & 0.066 \\
\hline Histological grade $(\mathrm{G} 1 / \mathrm{G} 2+\mathrm{G} 3)$ & 1.225 & 0.560 & 1.437 & 0.346 \\
\hline Tiam1 expression (high/low) & 0.266 & $<0.001$ & 0.401 & 0.020 \\
\hline
\end{tabular}

P-value in bold were statistically significant.
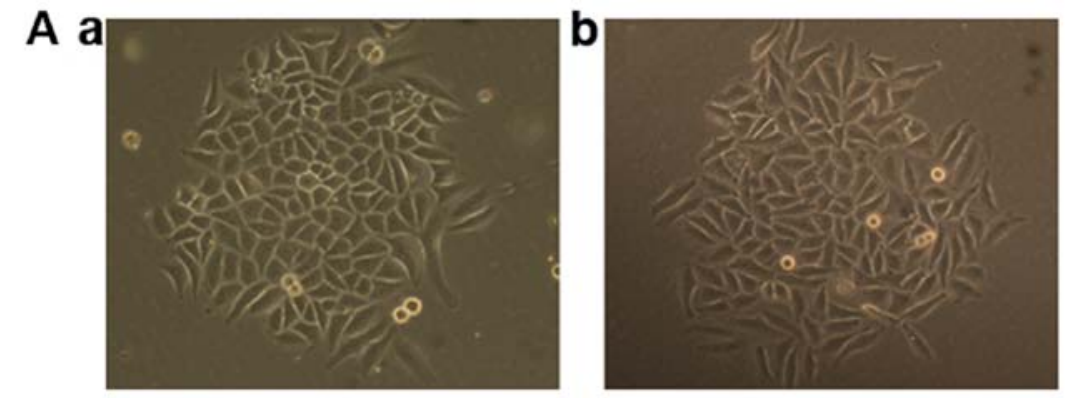

B
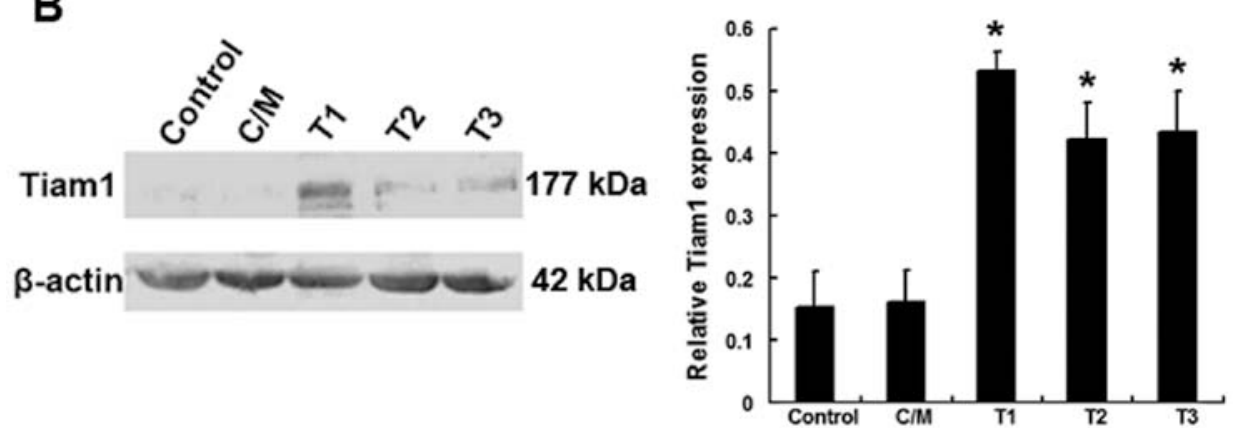

Figure 3. Overexpression of Tiam1 by transfection of Tiam1/C1199 plasmid in Hep-2 cell line. (A) Positive and negative cell clones that transfected different plasmid were selected by G418 (magnification, x100). (a, Negative cell clones; b, positive cell clones). (B) Upregulation of Tiam1 detected by western blotting. ( ${ }^{*} \mathrm{P}<0.001, \mathrm{~T} 1, \mathrm{~T} 2$, T3 vs. control, C/M) (control, Hep-2 cell group; C/M, Hep-2/Mock cell group; T1, T2 and T3, Hep-2/Tiam1 cell group). Tiam1, T-lymphoma invasion and metastasis-inducing factor 1 .

3 weeks. We randomly selected three positive cell clones that were transfected with Tiam1/C1199 cDNA (T1, T2 and T3) plasmid, and one negative cell clones that was transfected with vector control $(\mathrm{C} / \mathrm{M})$. Western blotting was carried out to assess the efficiency in the upregulation of Tiam1 expression. As shown in Fig. 3, Tiam1/C1199 cDNA was successfully transfected into the LSCC cells, and the level of Tiam1 expression was obviously upregulated $(\mathrm{P}<0.001)$. Moreover, we selected $\mathrm{T} 1$ cell clones for the subsequent experiments in vitro. The Hep-2 cells-transfected Tiam1/C1199 cDNA plasmid and vector control were termed, for convenience, as Hep-2/Tiam1 and Hep-2/Mock, respectively.

Upregulated Tiaml expression has no effect on proliferation of Hep-2 cells in vitro. To determine the effect of Tiam1 on the proliferation of Hep-2 cells in vitro, an MTT assay was performed and a cell growth curve was obtained. As shown in Fig. 4, no difference in cell growth between Hep-2/Tiam1 and Hep-2 and Hep-2/Mock control groups was identified ( $\mathrm{P}>0.05)$.

Upregulated Tiaml expression has no effect on cell cycle but decreases apoptotic rate of Hep-2 cells in vitro. To further elucidate the effect of upregulated Tiam1 on cell growth in Hep-2 cells, flow cytometry was carried out to monitor the cell cycle and apoptotic changes. The results demonstrated no difference between Hep-2/Tiam1 and the Hep-2 and Hep-2/Mock control groups in the G1 phase of the cell cycle $(66.02 \pm 3.48$ vs. $66.74 \pm 1.54,67.00 \pm 2.22)(\mathrm{P}>0.05)$, in the $\mathrm{S}$ phase of the cell cycle $(21.15 \pm 1.50$ vs. $19.65 \pm 1.61,17.83 \pm 3.63)$ $(\mathrm{P}>0.05)$ and in the $\mathrm{G} 2$ phase of the cell cycle (11.82 \pm 3.92 vs. 


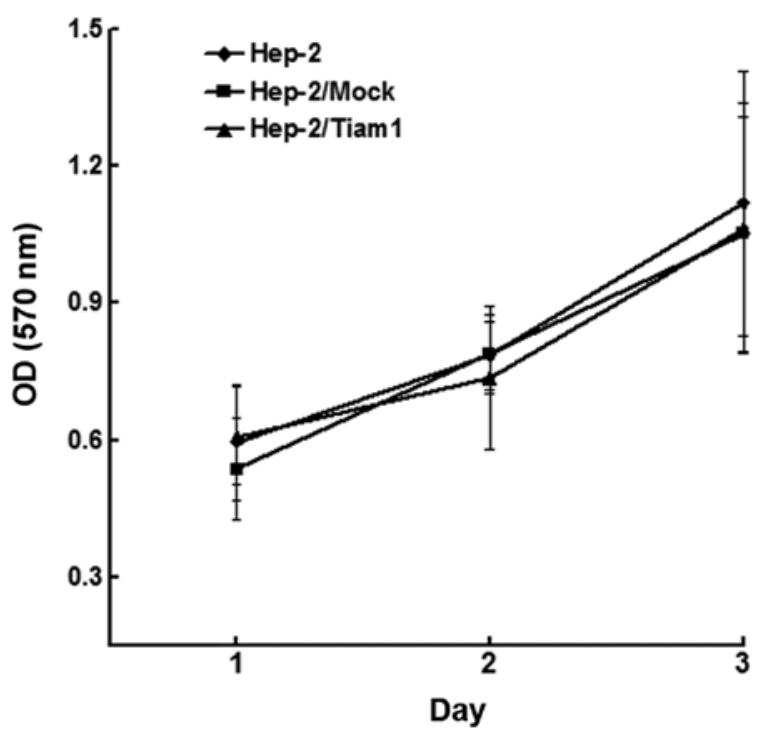

Figure 4. Effect of Tiam1 overexpression on the proliferation of Hep-2 cells. Cell proliferation was measured by the MTT assay every $24 \mathrm{~h}$ for 3 days. Tiam1, T-lymphoma invasion and metastasis-inducing factor 1.

13.66 $\pm 2.08,15.40 \pm 1.50)(\mathrm{P}>0.05)$ (Fig. 5A and B). However, when compared with the control groups, the apoptotic rate of Hep-2/Tiam1 was significantly decreased $(2.50 \pm 1.33$ vs. $5.61 \pm 1.08,5.83 \pm 1.02)(\mathrm{P}=0.021)($ Fig. 5C and D). These results demonstrated that upregulated Tiam1 expression has no effect on cell growth, but decreases the apoptotic rate of Hep- 2 cells.

Upregulated Tiaml expression promotes the migration and invasion of Hep- 2 cells in vitro. To determine the effect of Tiam1 on Hep-2 cell migration and invasion, wound-healing assay and Transwell invasion chambers were performed. As shown in Fig. 6A and C, upregulated Tiam1 expression can lead to significantly increased migration (Hep-2/Tiam1 group, migration distance $147.00 \pm 26.06$ vs. Hep-2, Hep-2/Mock control groups, migration distance $83.33 \pm 23.18$ and $96.33 \pm 16.01$; $\mathrm{P}=0.028)$ and invasion $(68.33 \pm 6.66$ vs. $43.00 \pm 8.89$ and $45.00 \pm 7.55 ; \mathrm{P}=0.013)$ of Hep- 2 cells. These results clearly demonstrate that Tiam1 plays a functional role in mediating cell migration and invasion in LSCC, which are also the key factors of LSCC malignant progression and metastasis.

\section{Discussion}

The oncogenic potential of Tiam1, the specific GEF for the Rho-like GTP-binding proteins, is achieved by the activation of Rac signaling pathway (18). Tiam1-Rac signaling is capable of stimulating the p38 mitogen-activated protein kinase pathway, c-Jun N-terminal kinase pathway, and extracellular signal-regulated kinase pathway (19-21), which are considered to be associated with the regulation of gene transcription. Tiam1 is involved in modulating alteration of the actin cytoskeleton, cell migration (22-24), tumor microenvironment (25) and cell polarity (26), which are considered relevant to tumor progression.

The relationship between Tiam 1 overexpression and various types of cancer has been previously identified (7-14).
However, to the best of our knowledge, few studies focusing on LSCC are available. Consequently, in the present study, we examined Tiam1 expression in LSCC tissue samples and assessed its clinical prognostic value. Furthermore, the role of Tiam 1 in the proliferation, apoptosis, migration and invasion of LSCC was elucidated in vitro.

In the present study, the results of 98 LSCC tissue samples investigated by immunohistochemistry demonstrated that a high Tiam1 expression was associated with lymph-node metastasis, recurrence, histological grade and clinical stage, which indicated the importance of Tiam1 in LSCC progression and metastasis. Additionally, the results of the present study show that Tiam1 overexpression was correlated with shorter disease-free and overall survival, and that Tiam1 may provide independent information to predict the clinical outcome of LSCC patients. The results of the Cox analysis revealed that Tiam1 expression and lymph-node metastasis were significant and independent prognostic factors involved in overall survival.

In the subsequent experiments, Tiam1/C1199 plasmid was successfully transfected to upregulate the expression of Tiam1 in LSCC cells. The MTT cell viability assay revealed that there was no significant effect on cell proliferation when LSCC cells were transfected with Tiam1/C1199 plasmid. Cell cycle analysis by flow cytometry also revealed that there was no difference in the G1, S and G2 phase of the cell cycle in the transfected cells. These results indicated that the upregulation of Tiam1 had no effect on the proliferation of LSCC cells. As mentioned in the immunohistochemistry results, Tiam1 overexpression was not associated with T stage of LSCC patients. Thus, the results of the MTT assay and cell cycle analysis in vitro are in concordance with the results obtained from immunohistochemistry. However, the critical role of Tiam1 in the proliferation of LSCC cells is discordant with its suggested roles in other reported cancers, such as colorectal cancer and hepatocellular carcinoma $(27,28)$. The discrepancy in these results is associated with the cell type-specific of Tiam1 in cell proliferation.

Cell migration and invasion play critical roles in cancer metastasis. Our in vitro data clearly indicated that upregulation of the Tiam1 expression increased the ability of migration and invasion of LSCC cells. Moreover, our results are in concordance with Tiam1 as identified in other reported cancers (27-29). Anoikis is reported to act as a physiological barrier to metastasis, however, resistance to anoikis may allow survival of cancer cells during metastasis (30). Cell apoptosis by flow cytometry in the present study showed that the apoptotic rate was decreased when LSCC cells were transfected with Tiam1/C1199 plasmid. Thus, we suggest that upregulation of Tiam1 expression can increase the ability of metastasis through a decrease of the LSCC cell anoikis rate. However, the underlying mechanisms regarding how Tiam1 regulates tumor metastasis have yet to be adequately elucidated.

In summary, results of the present study have demonstrated that the overexpression of Tiam1 was common in LSCC, which suggested that Tiam1 may play a critical role in inducing the tumorigenesis of LSCC. We also found that the upregulation of Tiam1 expression increased LSCC cell metastatic ability in vitro, suggesting that Tiam1 is a valuable therapeutic target for patients with LSCC. Future studies 
A

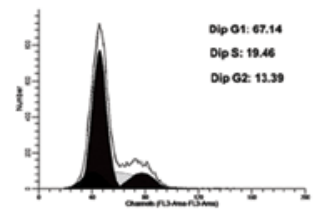

Hep-2

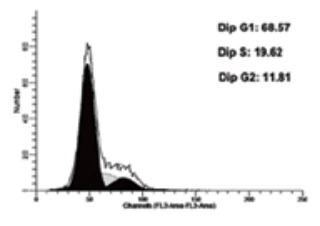

Hep-2/Mock
B

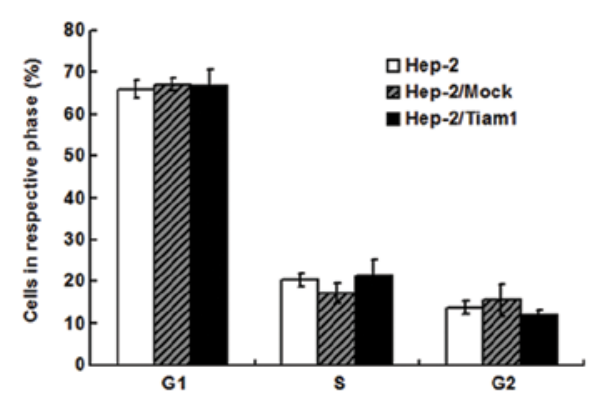

C

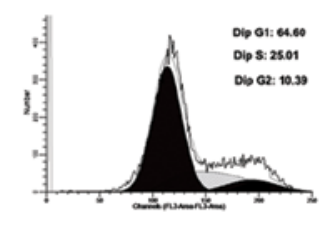

Hep-2/Tiam1

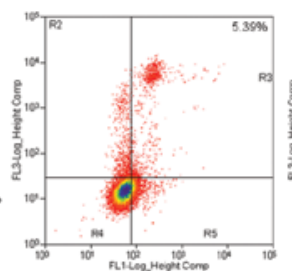

Hep-2

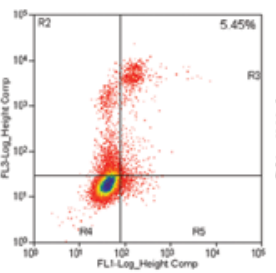

Hep-2/Mock

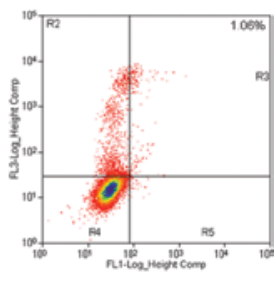

Hep-2/Tiam1
D

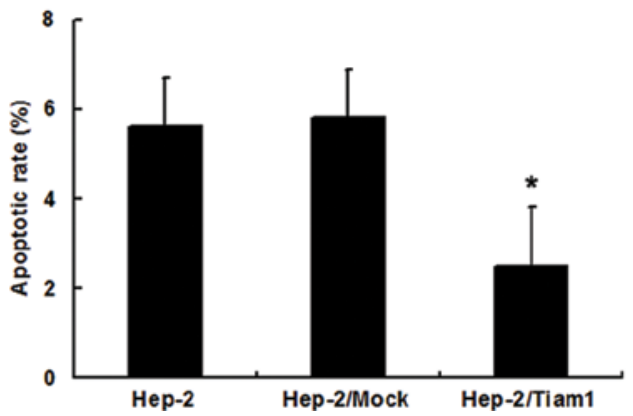

Figure 5. Effect of Tiam1 overexpression on cell cycle distribution and cell apoptosis of Hep-2 cells in vitro. (A) Representative histograms showing cell cycle profiles of Hep-2, Hep-2/Mock and Hep-2/Tiam1 cells. The cells were stained with propidium iodide (PI) and analyzed by flow cytometry. (B) Proportion of cells in various phases of cell cycle. (C) Representative histograms showing cell apoptosis of Hep-2, Hep-2/Mock and Hep-2/Tiam1 cells. Cells staining negative for PI but positive for Annexin V-FITC were considered to have undergone apoptosis. (D) Different apoptotic rates of various groups. ("P<0.05, Hep- $2 /$ Tiam1 vs. Hep-2, Hep-2/Mock). Tiam1, T-lymphoma invasion and metastasis-inducing factor 1.

A

$\mathrm{Oh}$

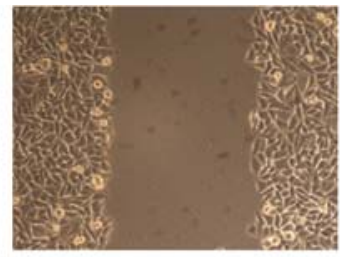

$48 \mathrm{~h}$

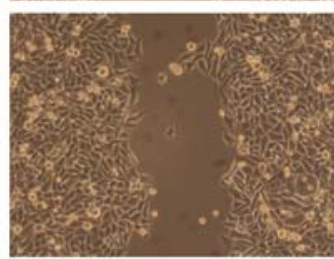

Hep-2

C

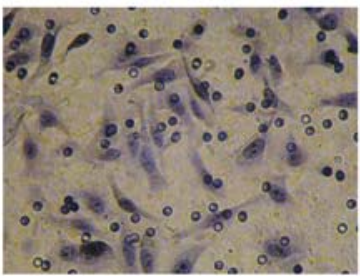

Hep-2
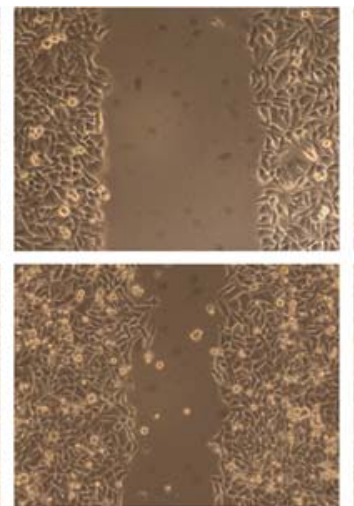

Hep-2/Mock

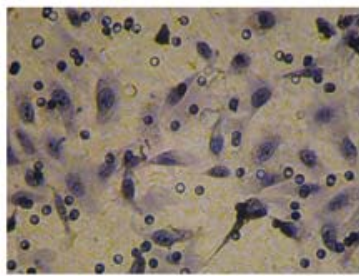

Hep-2/Mock
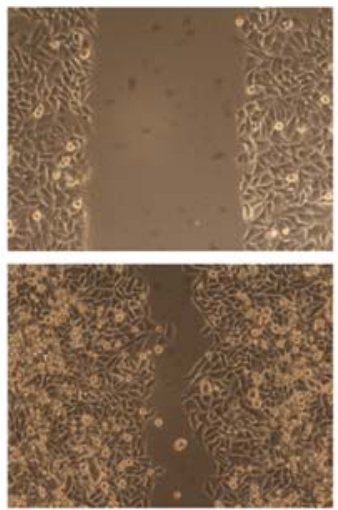

Hep-2/Tiam1

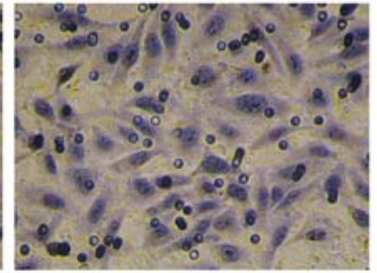

Hep-2/Tiam1

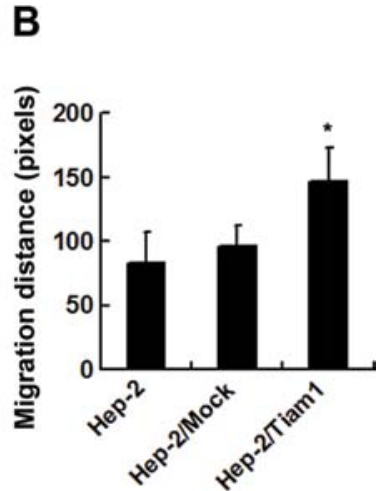

D

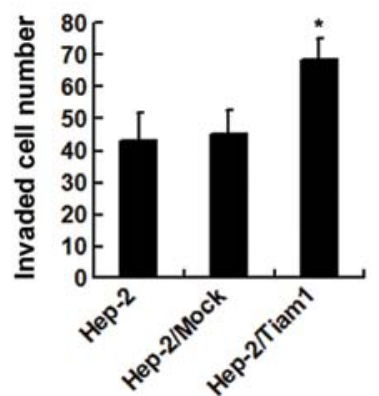

Figure 6. Effect of Tiam1 overexpression on migration and invasion of Hep-2 cells in vitro. (A) Wound-healing assay demonstrated that the overexpression of Tiam1 promoted the migration of Hep- 2 cells. Images were captured at 0 and $48 \mathrm{~h}$ after wound induction at x100 magnification. (B) Graphical representation of the distance of migration of Hep-2 cells. (C) Transwell invasion assay showed that overexpression of Tiam1 promoted the invasion of Hep-2 cells. Images were captured at x200 magnification. (D) Graphical representation of the numbers of invaded Hep-2 cells/microscopic field. ("P $<0.05$, Hep-2/Tiam1 vs. Hep-2, Hep-2/Mock). Tiam1, T-lymphoma invasion and metastasis-inducing factor 1.

should focus on the effect of Tiam1 overexpression in vivo and the precise mechanisms of Tiam1 in metastasis, which may lead to the identification of new techniques for the prevention of the progression of LSCC patients. 


\section{Acknowledgements}

We would like to thank Daiqiang Li and Songqing Fan (Department of Pathology, Second Xiangya Hospital, Central South University), and Yunjun Deng (Central Laboratory of Medical Research, Second Xiangya Hospital, Central South University) for their evaluation of the clinical samples. Dr Xinming Yang takes responsibility for the integrity of the content of the present study.

\section{References}

1. Sanderson RJ and Ironside JA: Squamous cell carcinomas of the head and neck. BMJ 325: 822-827, 2002.

2. Karatzanis AD, Psychogios G, Zenk J, Waldfahrer F, Hornung J, Velegrakis GA and Iro H: Comparison among different available surgical approaches in T1 glottic cancer. Laryngoscope 119: 1704-1708, 2009.

3. Cosetti M, Yu GP and Schantz SP: Five-year survival rates and time trends of laryngeal cancer in the US population. Arch Otolaryngol Head Neck Surg 134: 370-379, 2008.

4. Habets GG, Scholtes EH, Zuydgeest D, van der Kammen RA, Stam JC, Berns A and Collard JG: Identification of an invasion-inducing gene, Tiam-1, that encodes a protein with homology to GDP-GTP exchangers for Rho-like proteins. Cell 77: 537-549, 1994.

5. Mertens AE, Roovers RC and Collard JG: Regulation of Tiam1-Rac signalling. FEBS Lett 546: 11-16, 2003.

6. Minard ME, Kim LS, Price JE and Gallick GE: The role of the guanine nucleotide exchange factor Tiam1 in cellular migration, invasion, adhesion and tumor progression. Breast Cancer Res Treat 84: 21-32, 2004.

7. Engers R, Mueller M, Walter A, Collard JG, Willers R and Gabbert HE: Prognostic relevance of Tiam1 protein expression in prostate carcinomas. Br J Cancer 95: 1081-1086, 2006.

8. Ding Y, Chen B, Wang S, Zhao L, Chen J, Ding Y, Chen L and Luo R: Overexpression of Tiam1 in hepatocellular carcinomas predicts poor prognosis of HCC patients. Int J Cancer 124: 653-658, 2009.

9. Yang W, Lv S, Liu X, Liu H, Yang W and Hu F: Up-regulation of Tiam1 and Rac1 correlates with poor prognosis in hepatocellular carcinoma. Jpn J Clin Oncol 40: 1053-1059, 2010.

10. Qi Y, Huang B, Yu L, Wang Q, Lan G and Zhang Q: Prognostic value of Tiam1 and Rac1 overexpression in nasopharyngeal carcinoma. ORL J Otorhinolaryngol Relat Spec 71: 163-171, 2009.

11. Liu H, Shi G, Liu X, Wu H, Fan Q and Wang X: Overexpression of Tiam1 predicts poor prognosis in patients with esophageal squamous cell carcinoma. Oncol Rep 25: 841-848, 2011.

12. Zhao L, Liu Y, Sun X, He M and Ding Y: Overexpression of $\mathrm{T}$ lymphoma invasion and metastasis 1 predict renal cell carcinoma metastasis and overall patient survival. J Cancer Res Clin Oncol 137: 393-398, 2011.

13. Jin H, Li T, Ding Y, Deng Y, Zhang W, Yang H, Zhou J, Liu C, Lin J and Ding Y: Methylation status of T-lymphoma invasion and metastasis 1 promoter and its overexpression in colorectal cancer. Hum Pathol 42: 541-551, 2011.
14. Du X, Wang S, Lu J, et al: Clinical value of Tiam1-Rac1 signaling in primary gallbladder carcinoma. Med Oncol 29: 1873-1878, 2012.

15. Cao-Hong, Shibayama-Imazu T, Masuda Y, Shinki T, Nakajo S and Nakaya K: Involvement of Tiam1 in apoptosis induced by bufalin in HeLa cells. Anticancer Res 27: 245-249, 2007.

16. Bourguignon LY, Zhu H, Shao L and Chen YW: Ankyrin-Tiam1 interaction promotes Racl signaling and metastatic breast tumor cell invasion and migration. J Cell Biol 150: 177-191, 2000.

17. Bourguignon LY, Zhu H, Shao L and Chen YW: CD44 interaction with Tiam1 promotes Rac1 signaling and hyaluronic acid-mediated breast tumor cell migration. J Biol Chem 275: 1829-1838, 2000

18. van Leeuwen FN, van der Kammen RA, Habets GG and Collard JG: Oncogenic activity of Tiam1 and Rac1 in NIH3T3 cells. Oncogene 11: 2215-2221, 1995.

19. Zhang S, Han J, Sells MA, Chernoff J, Knaus UG, Ulevitch RJ and Bokoch GM: Rho family GTPases regulate p38 mitogenactivated protein kinase through the downstream mediator Pak1. J Biol Chem 270: 23934-23936, 1995.

20. Coso OA, Chiariello M, Yu JC, Teramoto H, Crespo P, Xu N, Miki T and Gutkind JS: The small GTP-binding proteins Rac1 and $\mathrm{Cdc} 42$ regulate the activity of the JNK/SAPK signaling pathway. Cell 81: 1137-1146, 1995.

21. Frost JA, Xu S, Hutchison MR, Marcus S and Cobb MH: Actions of Rho family small $\mathrm{G}$ proteins and p21-activated protein kinases on mitogen-activated protein kinase family members. Mol Cell Biol 16: 3707-3713, 1996.

22. Hou M, Tan L, Wang X and Zhu YS: Antisense Tiam1 downregulates the invasiveness of $95 \mathrm{D}$ cells in vitro. Acta Biochim Biophys Sin 36: 537-540, 2004.

23. Connolly BA, Rice J, Feig LA and Buchsbaum RJ: Tiam1-IRSp53 complex formation directs specificity of rac-mediated actin cytoskeleton regulation. Mol Cell Biol 25: 4602-4614, 2005.

24. Cordo-Russo RI, Alaniz LD, Saccodossi N, Lompardía S, Blanco G, Alvarez E, García MG and Hajos SE: Hyaluronan induces migration of multidrug-resistant lymphoma cell lines in vitro through Tiam 1 activation by a PI3K-dependent mechanism. Leuk Res 34: 1525-1532, 2010.

25. Xu K, Rajagopal S, Klebba I, Dong S, Ji Y, Liu J, Kuperwasser C, Garlick JA, Naber SP and Buchsbaum RJ: The role of fibroblast Tiam1 in tumor cell invasion and metastasis. Oncogene 29: 6533-6542, 2010.

26. Mertens AE, Pegtel DM and Collard JG: Tiam1 takes PARt in cell polarity. Trends Cell Biol 16: 308-316, 2006.

27. Liu L,Zhang Q,Zhang Y, Wang S and Ding Y: Lentivirus-mediated silencing of Tiam1 gene influences multiple functions of a human colorectal cancer cell line. Neoplasia 8: 917-924, 2006.

28. Huang J, Ye X, Guan J, et al: Tiam1 is associated with hepatocellular carcinoma metastasis. Int J Cancer 132: 90-100, 2013.

29. Shi YL, Miao RZ, Cheng L, Guo XB, Yang B, Jing CQ, Zhang L, Jin $\mathrm{X}$ and Li LP: Up-regulation of T-lymphoma and metastasis gene 1 in gastric cancer and its involvement in cell invasion and migration. Chin Med J 126: 640-645, 2013.

30. Douma S, Van Laar T, Zevenhoven J, Meuwissen R, Van Garderen E and Peeper DS: Suppression of anoikis and induction of metastasis by the neurotrophic receptor TrkB. Nature 430: 1034-1039, 2004. 